\title{
Posterior Reversible Encephalopathy Syndrome Induced by Spinal Computerized Tomography Contrast Agent: A Case Report
}

\author{
Do-Hyung KIM*, Soo Jin Yoon, Sang Hyun Jang, Jae Guk KIM, Sung-YeonSohn, Han Na Choi, Jin Ok Kim and \\ SooJoo LEE
}

Department of Neurology, Eulji University Hospital, Korea

Submission: August 24, 2017; Published: October 17, 2017

*Corresponding author: Do-Hyung KIM, Department of Neurology, Eulji University Hospital, School of Medicine, Eulji University, DunsanSeo-ro 95, Seo-gu, Daejeon 35233, Republic of Korea, Tel: +82-42-611-3431; Fax: +82-42-611-3858; Email: semoxx@naver.com

\begin{abstract}
A 58-year-old woman was admitted to the hospital for vertigo and ataxia that had developed after a CT scan was taken for spinal surgery 3 months before admission. In the fluid-attenuated inversion recovery and T2 weighted brain MRI scans that were performed at admission, a wide, symmetrical multifocal lesion with a high intensity signal was observed in both brain hemispheres, the external capsules, thalamus, white matter and sub cortical white matter around the cerebral ventricle of the frontal, parietal and temporal lobes. MRI taken at the time of disease onset, three months prior to the visit, confirmed the presence of mild posterior reversible encephalopathy syndrome. The patient was then admitted to the hospital for conservative treatment and later discharged. One month later, a follow-up brain MRI showed a slight decrease in signal intensity with a particularly more decrease at the brainstem. Hence, this case is reported to be a result of posterior reversible encephalopathy syndrome that occurred after the use of the CT contrast agent.
\end{abstract}

Keywords: Posterior reversible encephalopathy syndrome; CT contrast agent; Brain stem edema

Abbreviations: FLAIR: Fluid Attenuated Inversion Recovery; PRES: Posterior Reversible Encephalopathy Syndrome

\section{Introduction}

PRES, also referred to as reversible posterior leukoencephalopathy syndrome with hypertensive encephalopathy, usually occurs when a brain edema invades the parietooccipital region of the cerebrum and the posterior frontal cortical and sub cortical regions of the brain. When the edema progresses, it can be observed via imaging studies in the periventricular white matter, basal ganglia, brainstem, thalamus, internal capsules, anterior frontal lobe and parietotemporal cortex. Involvement of the basal ganglia and brain stem is uncommon, only occurring in $10-20 \%$ of patients [1]. Symptoms related to posterior cerebral blood circulation such as headaches, confusion, convulsions, and visual disturbances are commonly observed and often intensify in the short term [2]. PRES is known to be associated with acute and chronic renal disease, hypertension, blood vessel and autoimmune diseases, immunosuppressive therapies and medication, blood transfusions, organ transplants, infection, and anticancer drugs [3]. However, there are currently no reports of PRES onset following administration of a CT contrast agent. Here, we illustrate such a case, where a patient was admitted to hospital for vertigo and ataxia and diagnosed with PRES brought on by administration of a CT contrast agent.

\section{Case Report}

A 58-year-old female patient was admitted to the neurology department for dizziness. 3 months prior to admission, she had suffered a fall, incurring a lumbar fracture. At that time she was admitted to an external orthopedic hospital and underwent a lumbar CT with a contrast agent for an operation. The patient entered a state of confusion immediately after injection of the contrast agent. She had no memory for approximately one day, and although her memory began to return, she experienced dizziness that worsened over time. Dizziness was expressed not as vertiginous, but as ataxia. Symptoms worsened in the dark, and the patient commented that she felt as if her body was floating. With the onset of dizziness, the patient felt also that her vision was deteriorating, but investigation by the ophthalmology department found no abnormalities. She has no prior medical 


\section{Open Access Journal of Neurology \& Neurosurgery}

or family history aside from a hysteromyomectomy6 years ago. At the present admission, her blood pressure was $109 / 67 \mathrm{mmHg}$ with a pulse of 97 beats per minute. Body temperature and physical examinations were normal; however, she fell to both sides during the Tandem Gait test, and the Romberg test showed severe shaking on both sides. She reacted sensitively to questions asked during the interview and neurological examination and repeatedly asked questions herself. The results of the finger nose test and the finger to finger test were normal, but the heel-kneeshin test showed slight impairment in both legs. No dysarthria was observed and the Frenzel glasses test showed no sign of nystagmus. Muscular strength and sensory function in both upper and lower limbs were normal. Neither Babinski's sign nor Chaddock's sign were present. From serum analysis, complete blood count, electrolytes, liver function test, renal function test (BUN: 12, Cr: 0.63, eGFR: 98.7), diabetic screening, thyroid function, antinuclear antibodies including rheumatoid factor, electrophoresis, anti-human immunodeficiency virus antibody test, cytomegalovirus, herpes simplex virus, and Epstein-Barr virus antibody test were all normal. The results of the urine test were also normal. Brain MRI was performed to detect chronic cerebellar lesions. FLAIR and T2 weighted images showed a high signal intensity denoting a wide, symmetrical multifocal lesion across both brain hemispheres, the pons, external capsules, thalamus, white matter and sub cortical white matter around the ventricle of frontoparietotemporallobes (Figure 1). However, the diffusion weighted image, apparent diffusion coefficient, and T1 weighted images were normal. The enhanced image and magnetic resonance angiographic images were also normal. We performed additional cerebrospinal fluid and serum autoantibody tests in order to differentiate a wide spectrum of enhancement in the brain white matter. The cerebrospinal fluid examination and anti-gangliosides antibodies were normal. Syphilis antigen, antibody test, oligoclonal band, antiaquaporin 4 antibody, paraneoplasticantibody (anti-Hu, Yo, $\mathrm{Ri}$, amphiphysin, CRMP5, Ma2/Ta), arylsulfatase A (result: 0.56 , normal range: $0.50 \sim 2.0 \mathrm{nmol} / \mathrm{min} / \mathrm{mg}$ protein), antineutrophil cytoplasmic antibody (ANCA), and immunoglobulin were all normal. Blood and cerebrospinal fluid culture tests were negative, but anti-Ro antibodies (result: 75, normal range: $0-25$ ) and anti-La antibodies (result: 42, normal range: $0-25$ ) had increased slightly. Throughout the admission period, blood glucose remained normal and systolic and diastolic blood pressures were also normal. The patient's history was reconfirmed and the brain MRI taken 3 months previously, at the time of altered consciousness resultant from contrast agent administration, was verified. This original MRI showed a wide, symmetrical signal increase in the left thalamus and bilaterally in the white matter around the frontoparietotemporal lobes. However, it was less intense than the results of imaging from the present investigation and the pons was normal, thus it was confirmed that there was no involvement of the cortical white matter (Figure 1).

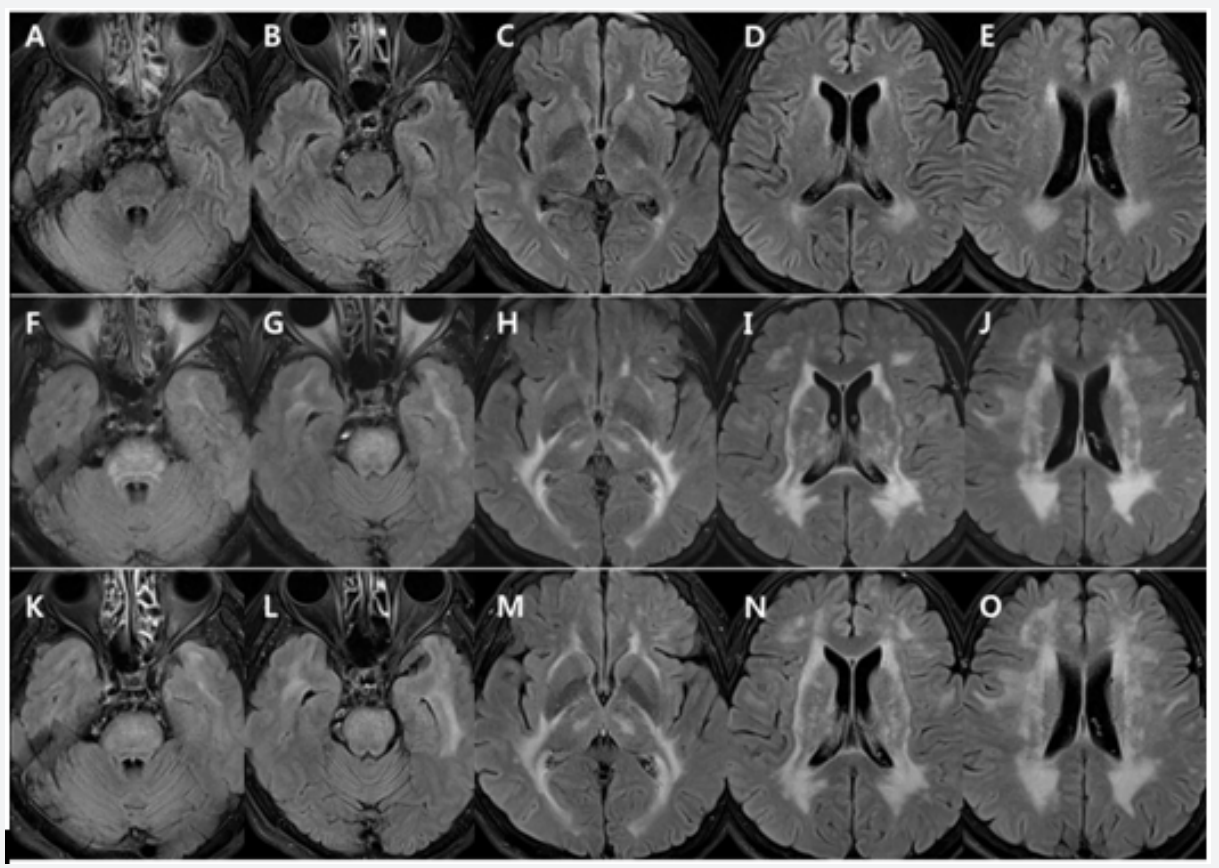

Figure 1: $(A)$ to $(E)$ show the MRI taken at the onset of symptoms that occurred after administration of the CT contrast agent, 3 months prior to admission to this hospital. Increased signal intensity was observed in the left thalamus and bilaterally in the white matter around the frontal, parietal and temporal lobes. $(F)$ to $(J)$ show the MRI taken at this hospital after admission. Signal intensity increases signify lesions in the periventricular white matter and sub cortical white matter of the pons, both external capsules, both thalamus, and both frontoparietotemporal lobes. $(\mathrm{K})$ to $(\mathrm{O})$ show the MRI taken as a follow-up exam 1 month after discharge. There is a slight decrease in signal intensity compared to the time of admission with further decreases seen in the pons. 


\section{Results}

The patient was diagnosed with PRES which resulted from the use of a contrast agent for CT. She was treated conservatively with intravenous normal saline for 2 weeks and blood pressure readings were confirmed to be stable. She was trained methods for preservation of renal function. The patient was re-admitted one month later without the administration of additional medication. She showed slight improvement in vertigo, but visual deterioration and gait instability persisted. In the neurological examination, Tandem Gait and Romberg test abnormalities were still apparent. A follow-up brain MRI showed a slight signal decrease in the pons compared with the time of admission, but the increased abnormal signal in the other areas remained (Figure 1).

\section{Discussion}

The patient in this case was diagnosed with PRES after brain MRI and additional tests ruled out a differential diagnosis of chronic posterior brain circulation abnormalities. Symptoms began with the onset of altered consciousness immediately after administration of a CT contrast agent which was performed prior to lumbar surgery three months ago. The brain MRI taken by a different hospital at the initial onset of symptoms also showed suggestions of PRES, thus leading to a PRES diagnosis resulting from CT contrast agent administration. Further, the signal intensity in the brain stem was successfully decreased through conservative treatment alone, also suggestive of PRES. Brain stem and basal ganglia involvement is generally uncommon in PRES, but brain edema occasionally appears in the brain stem and basal ganglia only, without edema in the typical brain regions for central-variant PRES. For PRES diagnosis, diseases that must be ruled out include rhombencephalitis, viral encephalitis, central pontinemyelinolysis, extrapontinemyelinolysis and basilar thrombosis with cerebral infarctions, tumefactive demyelination, and pontine glioma [1]. For this patient, these diseases were excluded through cerebrospinal fluid examination and brain imaging. Metachromatic leukodystrophy, which causes genetic alterations in the brain white matter, and arylsulfatase were also ruled out through the confirmation of negative results [4]. Damage to the blood-brain barrier has been linked to development of PRES due to high pressures that exceed the autoregulatory range and result in the release of plasma and macromolecules between blood vessels [2]. Since there are almost no sympathetic nerves in the posterior regions of the brain, these areas are particularly vulnerable to hyperperfusion and consequently, PRES. However, approximately 15-20\% of patients with PRES have normal or low blood pressure [2,5], and patients who present with fluctuating blood pressure levels may experience blood-brain barrier damage even at pressures within the normal range. With this in mind, the rapidity of blood pressure fluctuation appears to be more important than the exact blood pressure level. After the blood-brain barrier is damaged, presence of cytokines that increase the blood vessel wall permeability can cause interstitial brain edema, another factor related to the onset of PRES. The condition is also reported to be highly correlated with decreases in renal function $[2,3]$. Considering that internal endothelin increases after radio contrast administration, which in turn, results in increased blood vessel permeability [6], this patient experienced a rapid increase in cytokines after injection of the contrast agent that could have influenced blood vessel permeability in a similar manner to endothelin. This could have been a contributing factor in damaging the blood-brain barrier and causing brain edema. The contrast agent is believed to have temporarily decreased renal functionality thereby contributing to PRES development. However, the lesion shown by the MRI taken after admission to this hospital was wider and more serious compared to that seen when effects of the contrast agent first occurred. This could be a result of severe blood pressure fluctuations or repetition of transientrenal function degradation. Treatment of the illness was deferred due to a slight improvement seen in the follow-up brain MRI. The treatment was conservative in nature, focusing on stable blood pressure maintenance and training the patient to prevent renal function degradation during their period of hospitalization, since decreased renal function could have served as a cause of aggravation. Considering that clinical symptoms of PRES improved within one week of commencing treatment, with improvement verified through MRI [3], this patient is believed to have shown normal recovery expected after treatment of PRES.

While there are reports of PRES patients exhibiting different etiologies, the patient in this case is significant in that positive changes and progress were observed over time after the onset of her symptoms, which raises the possibility that a contrast agent for CT caused the condition. Brain edema in a patient with untreated, normal PRES may progress from the parietooccipital, posterior frontal cortical and sub cortical white matter to the brain stem. However, imaging tests confirm that when the cause of the disease is treated, the brain edema will improve in reverse order.

\section{Conflict of Interest}

All authors declare no conflicting interests and none of them are supported/funded by and drug company.

\section{References}

1. McKinney AM, Jagadeesan BD, Truwit CL (2013) Central-variant posterior reversible encephalopathy syndrome: brainstem or basal ganglia involvement lacking cortical or sub cortical cerebral edema. AJR Am J Roentgenol 201(3): 631-638.

2. Fugate JE, Rabinstein AA (2015) Posterior reversible encephalopathy syndrome: clinical and radiological manifestations, pathophysiology, and outstanding questions. Lancet Neurol 14(9): 914-925.

3. Hobson EV, Craven I, Blank SC (2012) Posterior reversible encephalopathy syndrome: a truly treatable neurologic illness. Perit Dial Int 32(6): 590-594.

4. Chavhan GB, Shroff MM (2009) Twenty classic signs in neuroradiology: A pictorial essay. Indian J Radiol Imaging 19(2): 135-145. 
5. Rabinstein AA, Mandrekar J, Merrell R, Kozak OS, Durosaro O, et al. (2012) Blood pressure fluctuations in posterior reversible encephalopathy syndrome. J Stroke Cerebrovasc Dis 21(4): 254-258.
6. Clark BA, Kim D, Epstein FH (1997) Endothelin and atrial natriuretic peptide levels following radiocontrast exposure in humans. Am J Kidney Dis 30(1): 82-86.

\section{Your next submission with Juniper Publishers will reach you the below assets}

- Quality Editorial service

- Swift Peer Review

- Reprints availability

- E-prints Service

- Manuscript Podcast for convenient understanding

- Global attainment for your research

- Manuscript accessibility in different formats ( Pdf, E-pub, Full Text, Audio)

- Unceasing customer service

Track the below URL for one-step submission https://juniperpublishers.com/online-submission.php 